\title{
Thermal Reversibility in Liquid-Liquid Phase Transition in Poly(vinyl ethylene-co-1,4-butadiene)/Polyisoprene Blends
}

\author{
Seiichi Kawahara and Saburo Akiyama \\ Laboratory of Chemistry, Faculty of General Education, \\ Tokyo University of Agriculture and Technology, \\ 3-5-8, Saiwaicho, Fuchu-shi, Tokyo 183, Japan
}

(Received June 14, 1989)

\begin{abstract}
For rubber blends of poly(vinyl ethylene-co-1,4-butadiene) with polyisoprene, reversible changes between phase dissolution and separation in the lower critical solution temperature (LCST) phase behavior were confirmed by differential scanning calorimetry. After the first annealing carried out to induce phase separation at a temperature above LCST, phase dissolution could be observed at the second annealing at a temperature $T<\mathrm{LCST}$, based on the liquid-liquid phase equilibrium. Observing phase transition from the double to the single $T_{\mathrm{g}}$ states, the phase dissolution time was found to be $6 \sim 379 \mathrm{~min}$. This dissolution time depends on both blend composition and annealing temperature. The apparent-activation energies of the phase dissolution were calculated to be $-3.6 \sim-17.34 \mathrm{kcal} \mathrm{mol}^{-1}$. The negative values of apparentactivation energies for the phase dissolution could be explained based on the equation of state.

KEY WORDS Thermal Reversibility/Miscibility/Vinyl Polybutadiene / Time/Glass Transition Behavior/Apparent-Activation Energy/LCST Phase Behavior /
\end{abstract}

In recent advanced studies on polymerpolymer blends, induced synergistic properties have been often attributed to their microstructures related to miscibility. Thus controlling miscibility or compatibility can play an important role in practical field. Recently physical $^{1-3}$ and morphological ${ }^{4}$ approaches have been widely investigated with respect to either miscible or immiscible states. The former is divided into two categories. One is thermodynamics and the other is kinetics. In the thermodynamic approach, it is useful to develop the concept of phase diagram. In practice, the phase behavior of polymer-polymer mixtures has been discussed in several literatures and reviews. ${ }^{5-10}$ On the other hand, the kinetic approaches have been investigated by mainly using small angle neutron scattering, ${ }^{11}$ light scattering ${ }^{12,13}$ and small angle $\mathrm{X}$-ray scattering. The origin of these ap- proaches is quite different; one is elucidated on equilibrium state, and the other is interpreted by the dynamics on non-equilibrium process. The phase diagram has, however, been depicted as a fundamental problem of both interpretations. In the thermodynamic process with phase behavior, a reversible change between homogeneous and heterogeneous states has been essential properties.

We carried out detailed studies on the phase reversibility of polymers, by dynamic mechanical loss measurements using torsional braid analysis (TBA), for a blend of poly(vinyl nitrate) (PVN) with poly(methyl acrylate) (PMA). ${ }^{14,15}$ Comparatively rapid phase dissolution behavior from initial two phases to one phase (the fastest phase dissolution time $\tau_{\mathrm{tr}}$ about $10 \mathrm{~min}$ ) was detected. Consequently, based on apparent-activation energies of phase dissolution, the segmental motion in the blend 
has been shown to the driving force for miscibility.

On the other hand, Vukovick and coworkers found that blends of poly(2,6-dimethyl-1,4-phenylene oxide) (PPO) with poly( $p$-fluorostyrene-co-o-fluorostyrene) ${ }^{16}$ and of PPO with poly(styrene-co-p-fluorostyrene), ${ }^{17}$ both of which showed double $T_{\mathrm{g}} \mathrm{s}$ on annealing, but exhibited single $T_{\mathrm{g}}$ upon cooling, by means of differential scanning calorimetry (DSC). Hasse and co-workers also reported the thermal reversibility of blends of chlorinated polybutadiene with chlorinated polyethylene. ${ }^{18}$ In addition, we also reported the reversibility of poly(vinylidine fluoride- $\mathrm{co}^{-}$ hexafluoro acetone) and poly(ethylene-co-vinyl acetate) blend. ${ }^{19}$ In this blend, a rapid change from opaque to transparent was detected through photometry.

In our recent study, ${ }^{20}$ a random copolymer, poly(vinyl ethylene-co-1,4-butadiene) (V-BR) above $32.3 \mathrm{wt} \%$ vinyl ethylene (vinyl) content was found to be miscible with polyisoprene (IR). For V-BR(32.3), at just $32.3 \mathrm{wt} \%$ vinyl content, LCST type phase behavior was confirmed. Moreover, in spite of high molecular weights, reversible change between double $T_{\mathrm{g}}$ state and single one was also observed with a comparatively rapid time scale.

In this study, the phase reversibility was investigated for V-BR(32.3)/IR blends by means of DSC measurement. Through this customary
$T_{\mathrm{g}}$ method, ${ }^{9,16}$ phase dissolution times from the double $T_{\mathrm{g}}$ to single $T_{\mathrm{g}}$ states were determined. Assuming that an apparent diffusion coefficient of phase dissolution was inversely proportional to the phase dissolution time, we adopted the Arrhenius type plot and calculated the apparent-activation energies.

\section{EXPERIMENTAL}

The polymer samples used in this study were V-BR(32.3) ànd IR, supplied by Nippon Zeon Co., Ltd. IR (Nipol $2200 \quad M_{w}=730,000$, $\left.M_{w} / M_{n}=1.84\right)$ was commercial polymer, while V-BR(32.3) $\left(M_{w}=214,000, M_{w} / M_{n}=1.03\right)$ was prepared by polymerizing 1,3-butadiene in hexane or benzene with a $n$-butyllithium catalyst in combination with diglyme(diethylene glycol dimethyl ether) at $40^{\circ} \mathrm{C}$.

Blend samples were mixed at specified blend ratios. The samples were prepared by precipitation from $5 \mathrm{wt} \%$ toluene solutions in ethanol. The precipitated samples were dried under vacuum at $40^{\circ} \mathrm{C}$ for a week. As-cast films were prepared by casting the solution onto a cover glass (for microscopy). The cast films were also dried under vacuum at $40^{\circ} \mathrm{C}$ for a week.

The process of measuring the phase dissolution time is shown in Figure 1. The prepared sample was annealed at $100^{\circ} \mathrm{C}$ for $5 \mathrm{~h}$ under vacuum. This annealed sample showing double $T_{\mathrm{g}}$ in DSC thermogram was quenched into liq. $\mathrm{N}_{2}$. The quenched sample was an-

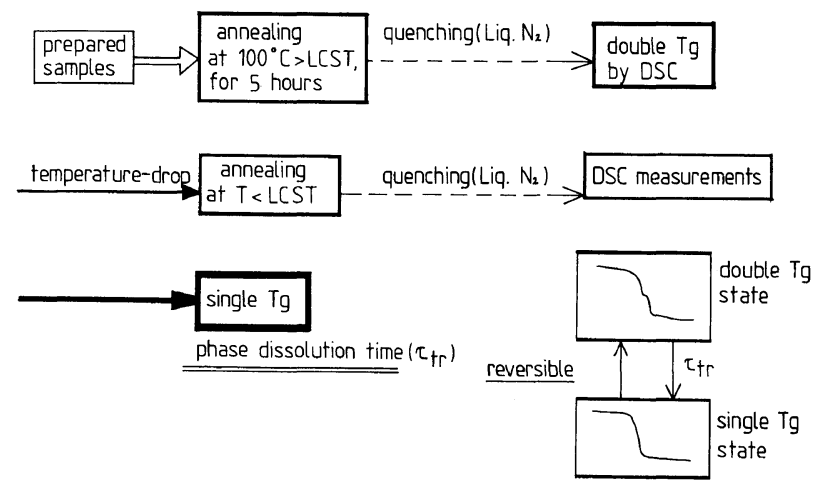

Figure 1. Thermal treatment procedure of the V-BR(32.3)/IR blend for determining phase transition time. 
nealed at a temperature lower than the phase boundary temperature in the LCST type phase diagram. The annealing time was set at about ten minutes intervals. In this procedure, the phase dissolution time was determined by single $T_{\mathrm{g}}$ in thermograms by the method shown in Figure 1. More detailed analytical criterion for determining $\tau_{\mathrm{tr}}$ will be defined in next section.

\section{RESULTS AND DISCUSSION}

As reported previously, the LCST type phase diagram was observed in V-BR(32.3)/IR blend as shown in Figure 2. The blends annealed at lower temperatures exhibited single $T_{\mathrm{g}}$ behavior, whereas they showed double $T_{\mathrm{g}}$ behavior at higher temperatures. Reversible changes between the double and the single $T_{\mathrm{g}}$ states were found to take place at a comparatively rapid time scale. For high molecular weight polymers, an LCST type phase diagram would be expected, if strong interactions such as hydrogen bonding and dipole-dipole interactions occur between the both polymer species in the blend. However, in this V-BR/IR which consists only of carbon and hydrogen, specific interactions are hardly expected. Since only 1,3-butadiene monomer is synthesized to make V-BR(32.3), repulsion may not be expected between only two monomeric species in V-BR(32.3). It may be considered that there are certain stereo-structural contributions between IR and random copolymer to these rubbers miscibility. In order to detect interactions between IR and V-BR(32.3), the phase dissolution time after forming the phase separated structure and apparent-activation energies were evaluated. In Figure 2, the relation between the annealing temperature and phase boundary temperature for LCST phase behavior is shown, where the broken line is the annealing temperature for the phase separation and the solid line is the LCST type phase boundary temperature. After the single phase samples were annealed at $100^{\circ} \mathrm{C}$ (on the

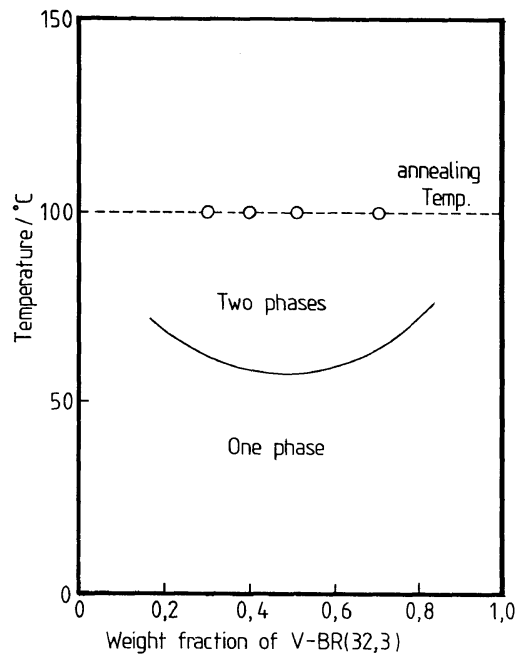

Figure 2. LCST type phase diagram of V-BR(32.3)/IR. The broken line is the annealing temperature for the phase separation.

broken line) for $5 \mathrm{~h}$, the temperature dropped below LCST. The phase dissolution phenomena could be observed at this second annealing temperature.

We could find comparatively rapid changes from double $T_{\mathrm{g}}$ to single one for LCST phase behavior. Then the phase dissolution times with a number of annealing experiments were determined. The phase dissolution time and the process have been shown relating to the phase separated structures annealed at $100^{\circ} \mathrm{C}, T>$ LCST. The phase separated structures of the cast films of V-BR(32.3)/IR blends through phase contrast microscope are shown in Figure 3. The small domains of both V-BR(32.3) and IR are clearly observed. Average domain size in each composition is shown in Table I. The domains are about $1 \mu \mathrm{m}$ in size. In accordance with the difference between a reflective index of V-BR(32.3) and IR, a bright domain is IR and a gloomy domain is $\mathrm{V}-\mathrm{BR}(32.3)$. When the blend ratio is $3 / 7$, the bright domain is larger than the gloomy domain, whereas, $7 / 3$, the bright domain is smaller than the gloomy domain. It was also found that the $7 / 3$ blend has the largest domain among the four. 


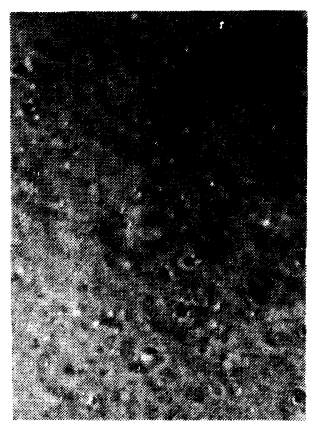

(a)

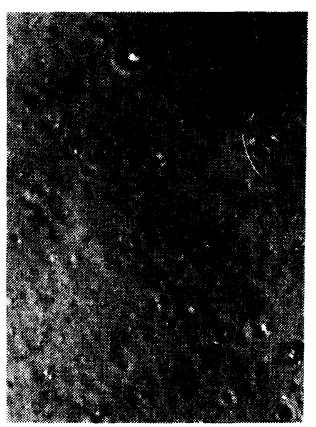

(b)

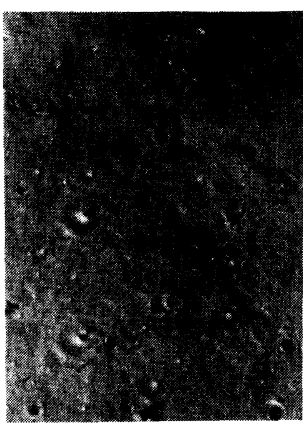

(c)

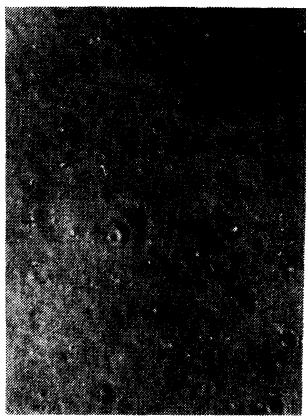

(d) $\overrightarrow{10 \mu \mathrm{m}}$

Figure 3. Light micrographs of V-BR(32.3)/IR blends at various blend ratios: (a), 7/3; (b), 5/5; (c), 4/6; (d), 3.7 .

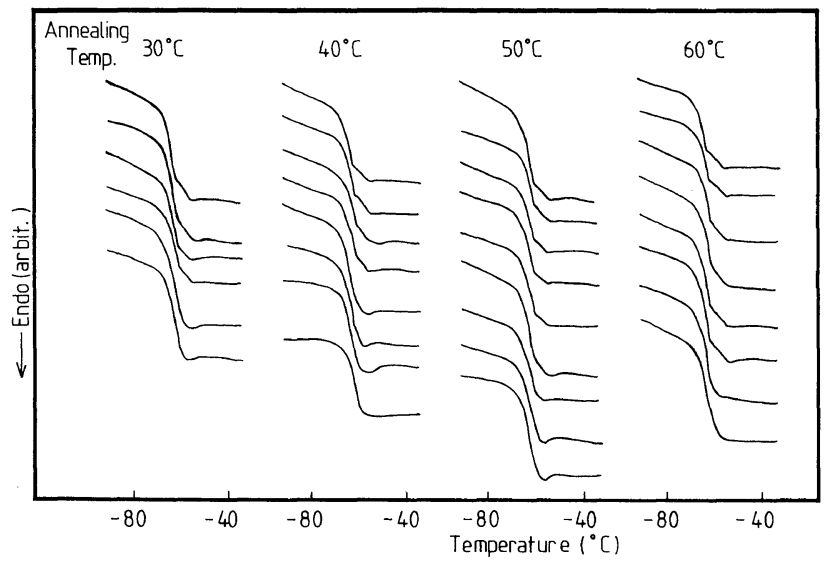

Figure4. Annealing temperature dependence of $T_{\mathrm{g}}$ behavior on DSC thermograms for V-BR(32.3)/IR $70 / 30$ blend, after setting adequate time intervals from top to bottom at each temperature.

Table I. Average domain size for V-BR(32.3)/IR blends with various compositions

\begin{tabular}{lcccc}
\hline & \multicolumn{4}{c}{ Domain size $/ \mu \mathrm{m}$} \\
\hline V-BR(32.3)/IR & $3 / 7$ & $4 / 6$ & $5 / 5$ & $7 / 3$ \\
\hline Bright domain & 1.29 & 0.89 & 1.28 & 2.13 \\
Gloomy domain & 1.10 & 0.70 & 1.04 & 2.66 \\
Average size & 1.20 & 0.80 & 1.16 & 2.40 \\
\hline
\end{tabular}

Figure 4 shows standard DSC thermograms for evaluating phase dissolution time. At each annealing temperature, the double $T_{\mathrm{g}}$ goes to a single one. A time to dissolve two phases to a homogeneous phase is the phase dissolution time $\left(\tau_{\mathrm{tr}}\right)$.

In order to obtain $\tau_{\mathrm{tr}}$ quantitatively, the deviation ratio is defined as following.

$$
\begin{aligned}
& \text { deviation ratio }(\%)= \\
& \frac{T_{\mathrm{g}, \text { bottom,blend }}-T_{\mathrm{g}, \text { top,blend }}}{T_{\mathrm{g}, \text { bottom, IR }}-T_{\mathrm{g}, \text { top }, \mathrm{V}-\mathrm{BR}}} \times 100
\end{aligned}
$$

where $T_{\mathrm{g}, \text { bottom,blend }}$ and $T_{\mathrm{g}, \text { top, blend }}$ are defined as the bottom and top points of intersection between the base line and tangent line at the inflection point of DSC thermograms for the blends, respectively, $T_{\mathrm{g} \text {,bottom, IR }}$ is as the bottom point of intersection of that for IR, and $T_{\mathrm{g}, \text { top, } \mathrm{V}-\mathrm{BR}}$ is as the top point intersection of that for V-BR(32.3), as shown in Figure 5. 


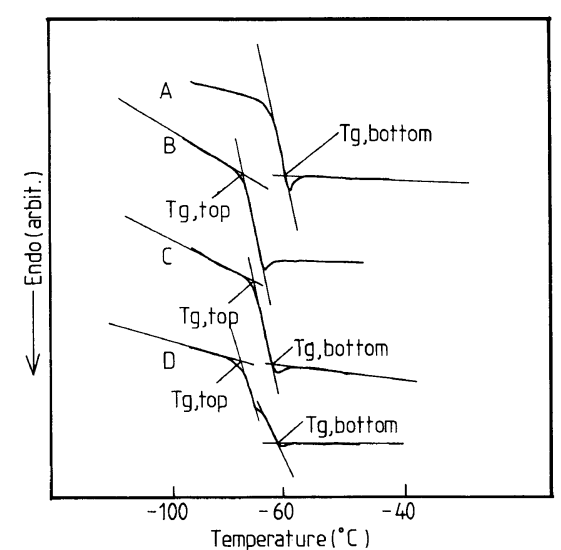

Figure 5. DSC thermograms of neat polymers and both single-phase and phase-separated samples in V-BR(32.3)/ IR blends. A, IR; B, V-BR(32.3); C, original single-phase blend; D, phase separated blend, annealed at $100^{\circ} \mathrm{C}$ for $2 \mathrm{~h}$.

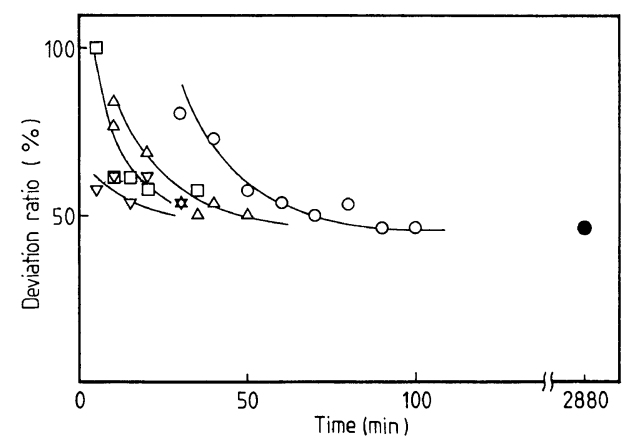

Figure 6. Blend $T_{\mathrm{g}}$ deviation from $T_{\mathrm{g}}$ of neat polymers, for V-BR(32.3)/IR 30/70 blend.

Since one of two $T_{\mathrm{g}} \mathrm{s}$ of phase separated blend annealed at $100^{\circ} \mathrm{C}$ for $5 \mathrm{~h}$ is very close to $T_{\mathrm{g}}$ of component polymer (thermogram $D$ ), the deviation ratio is $100 \%$. Figure 6 is a typical example of the deviation ratio of V-BR(32.3)/ IR (30/70). In the case of a single phase blend (thermogram $C$ ), the deviation ratio lies between 50 and $60 \%$ and is somewhat scattered. Since our experiment is assumed to be identical to a polymer relaxation method, $\tau_{\mathrm{tr}}$ can be estimated by following eq 2

$$
\Delta X / \Delta X_{0}=\exp (-t / \tau)
$$

where $\Delta X$ is a relaxation function as following

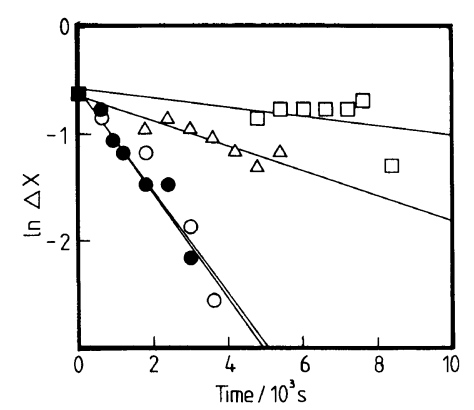

Figure 7. Semilogarithmic plot of $\Delta X$ vs. time $t$ in V-BR(32.3)/IR 7/3 blend: $(\bigcirc), 30 ;(\bigcirc), 40 ;(\triangle), 50 ;(\square)$, $60^{\circ} \mathrm{C}$, respectively.

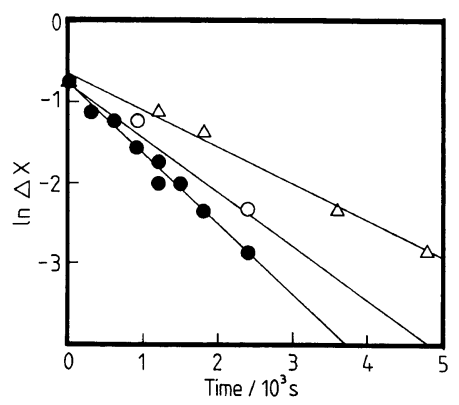

Figure 8. Semilogarithmic plot of $\Delta X$ vs. time $t$ in V-BR(32.3)/IR 5/5 blend: $(\bigcirc), 30 ;(\bigcirc), 40 ;(\triangle), 50^{\circ} \mathrm{C}$, respectively.

$$
\begin{aligned}
& \Delta X=X^{\infty}-X \\
& \Delta X_{0}=X^{\infty}-X_{0}
\end{aligned}
$$

It can be shown that $X_{0}$ is the deviation ratio of $100 \%$ as mentioned above, $X^{\infty}$ is the deviation ratio in single $T_{\mathrm{g}}$ state and $X$ is the deviation ratio of a time $t$. If a semilogarithmic plot of $\Delta X v s . t$ results in a linear relationship, its slope is expressed by $\left(\tau_{\mathrm{tr}}\right)^{-1}$. Figures 7 to 10 show semilogarithmic plots of $\Delta X v s . t$, in the $7 / 3,5 / 5,4 / 6$, and $3 / 7$ blends, respectively. When $t=0$, the straight lines go across $\Delta X_{0}$ owing to the definition.

According to above definition, the phase dissolution time was determined and was shown in Table II. At certain annealing temperatures, for example at $40^{\circ} \mathrm{C}$, the higher the V-BR(32.3) content in the blend is, the longer is phase dissolution time. When $T=40^{\circ} \mathrm{C}, \tau_{\mathrm{tr}}$ ranged 


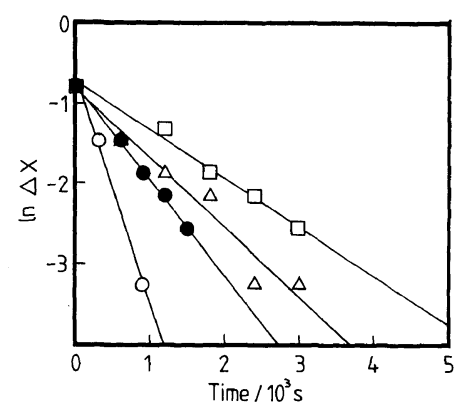

Figure 9. Semilogarithmic plot of $\Delta X$ vs. time $t$ in V-BR(32.3)/IR 4/6 blend: (O), 30; (๑), 40; $(\triangle), 50 ;(\square)$, $60^{\circ} \mathrm{C}$, respectively.

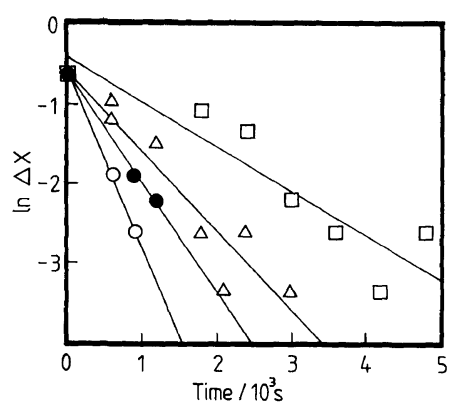

Figure 10. Semilogarithmic plot of $\Delta X$ vs. time $t$ in V-BR(32.3)/IR 3/7 blend: (O), 30; ( ), 40; $(\triangle), 50 ;(\square)$, $60^{\circ} \mathrm{C}$, respectively.

Table II. Phase dissolution time for V-BR(32.3)/IR blends

\begin{tabular}{crrrr}
\hline & \multicolumn{5}{c}{$\tau_{\text {tr }} / \mathrm{min}$} \\
\cline { 2 - 5 } Annealing temp. & \multicolumn{4}{c}{ V-BR(3.2)/IR } \\
\cline { 2 - 5 }${ }^{\circ} \mathrm{C}$ & $3 / 7$ & $4 / 6$ & $5 / 5$ & $7 / 3$ \\
\hline 30 & 8 & 6 & 25 & 33 \\
40 & 12 & 14 & 19 & 35 \\
50 & 17 & 19 & 36 & 143 \\
60 & 30 & 27 & - & 379 \\
\hline
\end{tabular}

from 12 to $35 \mathrm{~min}$. On the other hand, for the fixed blend ratio, the higher the annealing temperature the longer is the phase dissolution time is.

In order to interpret the phase dissolution behavior after phase separation, the apparentactivation energy of the phase dissolution is evaluated. Assuming that an apparent diffusion

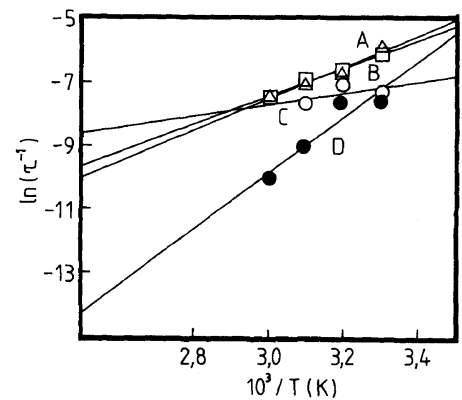

Figure 11. Arrhenius plot for phase dissolution. The blend ratios of $\mathrm{V}-\mathrm{BR}(32.3) / \mathrm{IR}$ are: A, 3/7; B, 4/6; C, 5/5; $\mathrm{D}, 7 / 3$, respectively.

Table III. Apparent-activation energies of phase dissolution for V-BR(32.3)/IR blends with various compositions

\begin{tabular}{cc}
\hline $\mathrm{V}-\mathrm{BR}(32.3) / \mathrm{IR}$ & $\Delta E_{\mathrm{app}} / \mathrm{kcal} \mathrm{mol}^{-1}$ \\
\hline $3 / 7$ & -8.77 \\
$4 / 6$ & -9.86 \\
$5 / 5$ & -3.60 \\
$7 / 3$ & -17.34 \\
\hline
\end{tabular}

coefficient of the phase dissolution is inversely proportional to the phase dissolution time, we calculated the apparent-activation energy by

$$
\log D_{\mathrm{app}}=\Delta E_{\mathrm{app}} / 2.303 R T+\log A
$$

where $\Delta E_{\mathrm{app}}$ is the apparent-activation energy, $A$ is the frequency factor, which has the same dimension of diffusion coefficient, and $D_{a p p}$ is the apparent diffusion coefficient of phase dissolution as following

$$
D_{\text {app }}=L^{2} / \tau
$$

where $L$ is the domain size considering the diffusion length and $\tau$ is the phase dissolution time corresponding the life time.

If a semilogarithmic plot of $D_{\text {app }} v s .1 / T$ is made, one can find that the terms of domain size are included in $\log A$ as a constant. Figure 5 shows a plot of $\log \left(\tau^{-1}\right)$ against reciprocal temperature. The apparent-activation energies can be determined from the slope of the least square mean equations in Figure 11. These results are shown in Table III. All $\Delta E_{\mathrm{qpp}}$ have 
negative values. Let us consider the negative values of $\Delta E_{\mathrm{app}}$ according to the customary interpretation of equation of state effects of phase transition. ${ }^{22-26}$

A difference in liquid structure (free volume) of the components can give rise to a negative contribution to excess enthalpy, entropy and volume, with a net effect which favor phase separation. Such effect on the equation of state is enhanced by increase in temperature. The competing effects of combinatorial entropy and differences in liquid structure can in principle give rise to both UCST and LCST. Prospects for the occurrence of such effect are limited by the requirement that the former be above the glass transition and the latter, below the decomposition temperature. In our case, only LCST can be found in an observable temperature range because of the lower UCST under the glass transition temperature. Trask and Roland ${ }^{27}$ recently reported that the LCST behavior for a blend of polybutadiene containing $8 \%$ 1,2-units at intermediate molecular weight with polyisoprene could be explained by the equation of state theory. Since the phase transition in V-BR(32.3)/IR blend may be explained by the equation of state effects, a driving force of phase dissolution can be enhanced with increasing the departure from LCST in temperature dropping for annealing. A magnitude of difference between the chemical potential of mixing at $100^{\circ} \mathrm{C}$ and that of the phase dissolution temperature $T<$ LCST, which is one of the driving forces of phase dissolution, increases with decreasing temperature. Then, below LCST, the phase dissolution time becomes short with decreasing annealing temperature above $T_{\mathrm{g}}$, so that the negative $\Delta E_{\text {app }}$ can be resulted.

Phase dissolution can be elucidated by balance between enthalpy and entropy of mixing.

$$
\begin{aligned}
\Delta G_{\mathrm{min}}= & \Delta H_{\mathrm{mix}}-T \Delta S_{\mathrm{mix}}=\Delta U_{\mathrm{mix}} \\
& +p \Delta V_{\mathrm{mix}}-T \Delta S_{\mathrm{mix}}
\end{aligned}
$$

Polym. J., Vol. 22, No. 5, 1990
As to the thermodynamics of non-equilibrium process, the rate of phase dissolution is interpreted by a departure from equilibrium condition. In the absence of strong interactions such as hydrogen bonding and dipole-dipole interactions between V-BR and IR, these two approaches may explain the presence of certain structures in stationary states at various temperatures. The phase dissolution can spontaneously occur up to an equilibrium state below LCST after annealing at $100^{\circ} \mathrm{C}$ for $5 \mathrm{~h}$. Then the phase dissolution time may be related to both the free volume and segmental motion as a function of temperature. A departure of annealing time from LCST is the major effect for dominanting the phase dissolution and separation processes. Recently, thermal reversibility in liquid-liquid phase transition in high molecular weight dissimilar polymer blends has been reported. ${ }^{28,29}$ Sato and Han carried out quantitative investigation on both sides of the phase boundary using small angle light scattering technique. The mobility $M$, extractable from the interdiffusion coefficient $D$ by combining the results of both statics and kinetics had an Arrhenius type of temperature dependence on both sides of the phase boundary. ${ }^{29}$ In our study, the phase dissolution behavior proved to show a similar temperature dependence of their result, although our interdiffusion process and free energy density were not evaluated yet.

In upper critical solution temperature (UCST) type phase behavior, when $T>$ UCST, the apparent activation energies for phase diffusion have been evaluated. ${ }^{21}$ In both UCST and LCST behavior, the driving force of phase dissolution can be interpreted by the equation of state effects, i.e., the segmental interaction, the free volume effect and the size effect. The segmental interaction is the main favorable effect just below the LCST for the phase dissolution. The strong specific interaction between V-BR(32.3) and IR is hardly predicted. Therefore, instead of specific interaction, certain favorable stereo-structures which 
should enhance miscibility for the V-BR(32.3)/ IR can be expected.

\section{CONCLUSIONS}

Reversible changes between double $T_{\mathrm{g}}$ states and single one were observed in LCST phase behavior of V-BR(32.3)/IR in spite of high molecular weights. The V-BR(32.3)/IR blends annealed at $100^{\circ} \mathrm{C}$ for $5 \mathrm{~h}$ (double $T_{\mathrm{g}}$ states) showed a single $T_{\mathrm{g}}$ when annealed below the phase boundary temperatures. By the $T_{\mathrm{g}}$ method, a phase transition time from 6 to $379 \mathrm{~min}$ was obtained. The apparent-activation energies were $-3.60 \sim-17.34 \mathrm{kcal} \mathrm{mol}^{-1}$ from the Arrhenius type expression. Based on apparent-activation energies, the phase dissolution process takes place spontaneously up to equilibrium state which is provided by the phase dissolution temperature, $T<$ LCST. Since the temperature is lower, the phase dissolution time is faster and the apparentactivation energy of phase dissolution takes on a negative value. Thus, there are any favorable structures which enhance the miscibility for the V-BR(32.3)/IR. More detailed thermodynamic investigations are being now conducted.

Acknowledgments. The authors express their sincere thanks to Dr. A. Ueda of Nippon Zeon Co., Ltd. for supplying the V-BR and IR.

\section{REFERENCES}

1. O. Olabishi, L. M. Robeson, and M. T. Shaw, "Polymer-Polymer Miscibility," Academic Press, New York, N.Y., 1979.

2. S. Akiyama, T. Inoue, and T. Nishi, "Polymer Blends-Compatibility and Interface" (in Japanese), CMC Press, Tokyo, 1979 (R\&D report) and 1981.

3. D. R. Paul and S. Newman (Ed.), "Polymer Blends,"
Vols. 1 and 2, Academic Press, New York, N.Y., 1978.

4. H. Tanaka, T. Hayashi, and T. Nishi, J. Appl. Phys., 65, 4480 (1989).

5. P. J. Flory, R. A. Orwall, and A. Vrij, J. Am. Chem. Sci., 86, 3507 (1964).

6. L. P. McMaster, Macromolecules, 6, 760 (1973).

7. I. C. Sanchez and R. H. Lacombe, J. Phys. Chem., 80, 2352 (1976).

8. D. Patterson and A. Robard, Macromolecules, 11, 690 (1978).

9. G. ten Brinke and F. E. Karasz, Macromolecules, 17, 815 (1984).

10. S. Akiyama, Hyomen, 23, 617 (1985).

11. M. Shibayama, H. Yang, R. S. Stein, and C. C. Han, Macromolecules, 18, 2089 (1985).

12. J. Maruta, T. Ougizawa, and T. Inoue, Polymer, 29, 2056 (1988).

13. P. G. de Gennes, "Scaling Concepts in Polymer Physics," Cornell University Press, New York, 1979.

14. S. Akiyama, I. Matsumoto, M. Nakata, and R. Kaneko, Kobunshi Ronbunshu, 33, 238 (1976).

15. S. Akiyama, Kagaku to Kogyo, 62, 382 (1988).

16. R. Vukovic, F. E. Karasz, and W. J. MacKnight, Polymer, 24, 529 (1983).

17. R. Vukovic, V. Kuresevic, F. E. Karasz, and W. J. MacKnight, Thermochim. Acta, 54, 349 (1982).

18. M. A. Hasse, H. Ueda, and F. E. Karasz, Macromolecules, 21, 3438 (1988).

19. M. Hasegawa and S. Akiyama, Polym. J., 20, 471 (1988).

20. S. Kawahara, S. Akiyama, and A. Ueda, Polym. J., 21, 221 (1989).

21. Y. Takagi, T. Ougizawa, and T. Inoue, Polymer, 28, 103 (1987).

22. K. S. Siow, G. Delmas, and D. Patterson, Macromolecules, 5, 29 (1972).

23. S. L. Zacharius, G. ten Brinke, W. J. MacKnight, and F. E. Karasz, Macromolecules, 16, 381 (1983).

24. C. T. Murray, J. W. Gilmer, and R. S. Stein, Macromolecules, 18, 996 (1985).

25. G. Cong, Y. Huang, W. J. MacKnight, and F. E. Karasz, Macromolecules, 19, 2765 (1986).

26. H. W. Kammer, T. Inoue, and T. Ougizawa, Polymer, 30, 888 (1989).

27. C. A. Trask and C. M. Roland, Polym. Commun., 29, 332 (1988).

28. G. R. Strobl, J. T. Bendler, R. P. Kambour, and A. R. Shultz, Macromolecules, 19, 2683 (1986).

29. T. Sato and C. C. Han, J. Chem. Phys., 88, 2057 (1988). 